PROCEEDINGS OF THE

AMERICAN MATHEMATICAL SOCIETY

Volume 132, Number 7, Pages 2151-2158

S 0002-9939(04)07304-6

Article electronically published on January 22, 2004

\title{
COUPLINGS OF UNIFORM SPANNING FORESTS
}

\author{
LEWIS BOWEN
}

(Communicated by Richard C. Bradley)

\begin{abstract}
We prove the existence of an automorphism-invariant coupling for the wired and the free uniform spanning forests on connected graphs with residually amenable automorphism groups.
\end{abstract}

\section{INTRODUCTION}

All the graphs in this paper will be assumed to be locally finite with at most a countable number of vertices. Given a (connected) graph $\Gamma$, there exist two natural random subgraphs on $\Gamma$ known as the Free Uniform Spanning Forest (FSF) and the Wired Uniform Spanning Forest (WSF) (see BLPS or [L1 for introductory material and more). They are defined as follows. Consider any increasing sequence $\Gamma_{i}$ of finite connected subgraphs of $\Gamma$ whose union is all of $\Gamma$. Then, as shown in $[\mathrm{Pe}$, the uniform distribution on spanning trees on $\Gamma_{i}$ converges and is, by definition, the distribution of FSF. To obtain WSF, identify all of the boundary vertices of $\Gamma_{i}$ to obtain a graph $\tilde{\Gamma}_{i}$. As proven in $[\mathrm{Pe}$, the uniform distribution on spanning trees on $\tilde{\Gamma}_{i}$ converges and the limiting distribution is the distribution of WSF (by definition).

In [BLPS, it is shown that there exists a monotone coupling $\mu$ of WSF and FSF (this follows from $[\mathrm{FM}]$ ); i.e., $\mu$ is a probability measure on $2^{E(\Gamma)} \times 2^{E(\Gamma)}$ (where $E(\Gamma)$ denotes the edge set of $\Gamma$ ) whose projection onto the first factor is the distribution of WSF, whose projection onto the second factor is the distribution of FSF, and such that $\mu$ is concentrated on the set of all pairs $\left(T_{1}, T_{2}\right) \in 2^{E(\Gamma)} \times 2^{E(\Gamma)}$ such that $T_{1} \subset$ $T_{2}$. The proof, however, is purely an existence proof. The authors of BLPS posed the following problem: does there exist a natural or explicit monotone coupling of WSF and FSF? In particular, does there exist an automorphism-invariant coupling of WSF and FSF?

We show that if $\Gamma$ is a (connected, locally finite) graph and $G<\operatorname{Aut}(\Gamma)$ is a residually amenable group of automorphisms of $\Gamma$, then there exists a coupling of WSF and FSF that is invariant under elements of $G$. We note that all finitely generated linear groups are residually amenable. See Section 2 for more details on residual amenability.

Received by the editors January 30, 2003 and, in revised form, April 14, 2003.

2000 Mathematics Subject Classification. Primary 60D05, 05C05, 60B99, 20 F32.

Key words and phrases. Spanning trees, Cayley graphs, couplings, harmonic Dirichlet functions, amenability, residual amenability.

This research was supported in part by NSF Vigre Grant No. DMS-0135345. 
We mention that for many graphs $\Gamma$, WSF $=\mathrm{FSF}$. This is true if and only if there are no nonconstant harmonic Dirichlet functions on $\Gamma$ (see [BLPS]). In particular, $\mathrm{WSF}=\mathrm{FSF}$ whenever $\Gamma$ is the Cayley graph of a cocompact lattice $G$ in $\mathbb{E}^{n}$ (for all $n$ ) or in $\mathbb{H}^{n}$ (for $n>2$ ). However, WSF $\neq \mathrm{FSF}$ when $G$ is a cocompact Fuchsian group.

\section{BACKGRound ON RESIDUALLY AMENABLE GROUPS}

We will use the following definition of amenable (see $[\mathbf{Z}]$ ):

A topological group $G$ is amenable if and only if for every compact metrizable space $Z$ such that $G$ acts on $Z$ by homeomorphisms and the map $G \rightarrow H$ omeo( $Z)$ is continuous, there exists a Borel probability measure $\mu$ on $Z$ that is invariant under the action of $G$. By invariant, we mean that for all Borel sets $E \subset Z$ and for all $g \in G, \mu(g E)=\mu(E)$. The topology on Homeo $(Z)$ is the compact open topology. We note that finite groups and finitely generated abelian groups are amenable (given the discrete topology).

If $P$ is a property of groups, then a topological group $G$ is said to be residually $P$ if for every $g \in G-\{1\}$, there exists a surjective continuous homomorphism $\phi: G \rightarrow H$ such that $H$ has property $P$ and $\phi(g)$ is not the identity.

We need the following two results from [Z], Prop. 4.1.6. First, if $G$ is amenable and $H$ is a closed subgroup of $G$, then $H$ is amenable. Second, if $K$ is a closed normal subgroup of $G$, then $G$ is amenable if and only if both $K$ and $G / K$ are amenable. Now suppose that $K_{1}$ and $K_{2}$ are closed normal subgroups of $G$ such that both $G / K_{1}$ and $G / K_{2}$ are amenable. We claim that $G /\left(K_{1} \cap K_{2}\right)$ is amenable. To see this, note that $K_{1} /\left(K_{1} \cap K_{2}\right)$ is a closed normal subgroup of $G /\left(K_{1} \cap K_{2}\right)$ and $\left[G /\left(K_{1} \cap K_{2}\right)\right] /\left[K_{1} /\left(K_{1} \cap K_{2}\right)\right]$ is isomorphic to $G / K_{1}$, which is amenable. Also, $K_{1} /\left(K_{1} \cap K_{2}\right)$ maps injectively into $G / K_{2}$ (under the quotient map); so it is amenable too (by the first result). The second result now implies that $G /\left(K_{1} \cap K_{2}\right)$ is itself amenable. It follows easily that if $G$ is separable (i.e., $G$ contains a countable dense subset), then $G$ is residually amenable if and only if there exists a decreasing sequence of closed normal subgroups $G_{i}$ in $G$ such that $G / G_{i}$ is amenable and $\bigcap_{i} G_{i}=\{1\}$. The next lemma is presumably well known, but we did not find it in the literature.

Lemma 2.1. If $\Gamma$ is a locally finite graph (with at most a countable number of vertices) and $G$ is a group of automorphisms of $\Gamma$, then $G$ is separable (in the open-compact topology).

Proof. Let $V$ denote the vertex set of $\Gamma$. We let $B_{r}(v)$ denote the radius $r$ neighborhood of a vertex $v \in V$. Since $\Gamma$ is locally finite, for every integer radius $r>0$ and $(v, w) \in V \times V$, there are only finitely many bijections from $B_{r}(v)$ to $B_{r}(w)$. So there exists a countable set $S \subset G$ such that for any $(v, w) \in V \times V$, if there is a $g \in G$ such that $g(v)=w$, then there exists a $g_{r} \in S$ that agrees with $g$ on the radius $r$ neighborhood of $v$. In the open-compact topology, $g_{r}$ converges to $g$ as $r \rightarrow \infty$. So $S$ is dense in $G$.

We let $\operatorname{Aut}(\Gamma)$ denote the full automorphism group of a graph $\Gamma$.

Corollary 2.2. If $G<A u t(\Gamma)$ is residually amenable, then there exists a decreasing sequence $\left\{G_{i}\right\}$ of closed normal subgroups of $G$ such that $G / G_{i}$ is amenable and $\bigcap_{i} G_{i}=\{1\}$. 
We note that since finite groups are amenable, any residually finite group is residually amenable. A well-known result due to Mal'cev [Ma (or see theorem 2.7 We states that if $G$ is a finitely generated subgroup of $G L_{n}(\mathbb{C})$, then $G$ is residually finite. In particular, all discrete groups of isometries of hyperbolic space are residually finite. It is an open problem whether or not all word hyperbolic groups are residually finite (see $[\mathrm{KW}]$ ). Olshanskii has proved [O] that in a certain sense, almost every finitely generated group is word hyperbolic. So, if all word hyperbolic groups are residually finite, then almost every (finitely generated) group is residually amenable.

There is an example, due to E. A. Scott $[\underline{S}]$ of a finitely generated infinite simple group that has a free nonabelian subgroup. Such a group cannot be residually amenable. There are groups that are residually amenable but not residually finite. For example, the Baumslag-Solitar group $B S(2,3)=\left\langle x, y \mid x y^{2} x^{-1}=y^{3}\right\rangle$ is one.

\section{BACKGROUND ON DETERMINANTAL PROBABILITY MEASURES}

Let $E$ be any set (with at most countable cardinality), and let $S<l^{2}(E)$ be a closed subspace of $l^{2}$-summable functions on $E$. (In general, we write $S<T$ to mean that $S$ is a closed subspace of $T$.) There exists a natural probability measure $\mathbf{P}^{S}$ on $2^{E}$ defined by

$$
\mathbf{P}^{S}(B \subset \mathfrak{B})=\operatorname{Det}\left[\left\langle P_{S}\left(\chi^{e}\right), \chi^{f}\right\rangle_{e, f \in B}\right] .
$$

$\mathbf{P}^{S}$ is said to be a determinantal probability measure (see L2 for the history and background of such measures). Here, $B$ is a finite subset of $E$ and $\mathbf{P}^{S}(B \subset \mathfrak{B})$ is, by definition, the probability with respect to $\mathbf{P}^{S}$ that $B$ is contained in a random subset of $\Gamma, P_{S}$ denotes projection onto $S, \chi^{e}$ denotes the characteristic function of an element $e \in B$. The right-hand side is the determinant of the matrix with columns and rows indexed by elements of $B$ whose $(e, f)$-entry is equal to the $l^{2}$ inner product $\left\langle P_{S}\left(\chi^{e}\right), \chi^{f}\right\rangle$. Using the inclusion-exclusion principle, it can be shown that this determines a probability measure. A determinantal probability measure is defined as one that arises from this construction (see [L2] for introductory material and more). We let $\mathcal{G}_{S}$ denote a random subset with distribution $\mathbf{P}^{S}$.

It is shown in [BLPS that the distributions of WSF and FSF are determinantal. To be precise, let $\Gamma$ be a graph. We let $l_{-}^{2}(\Gamma)$ be the space of $l^{2}$-summable antisymmetric functions $f$ on the (directed) edge set of $\Gamma$. By antisymmetric we mean that if $e$ is an edge of $\Gamma$ and $\check{e}$ is equal to $e$ with the opposite orientation, then $f(e)=-f(\check{e})$. For any edge $e$ of $\Gamma$, let $\mathbf{1}_{e}$ be the characteristic function of $e$. We let $\chi^{e}=\mathbf{1}_{e}-\mathbf{1}_{\check{e}}$ be the unit flow along the (directed) edge $e$.

For any vertex $v$ of $\Gamma$, define the star of $v$ to be $\Sigma_{e} \chi^{e}$ where the sum is over all directed edges $e$ with initial endpoint equal to $v$. We let $\star$ be the space in $l_{-}^{2}(\Gamma)$ generated by all stars of all vertices.

We say that $\left(e_{1}, \ldots, e_{k}\right)$ is a cycle of edges in $\Gamma$ if the destination vertex of $e_{i}$ is equal to the initial vertex of $e_{i+1}$ for all $i \bmod k$. In this case, we say that the function $\Sigma_{i} \chi^{e} \in l_{-}^{2}(\Gamma)$ is a cycle. We let $\diamond$ be the subspace of $l_{-}^{2}(\Gamma)$ generated by all cycles in $\Gamma$. It is shown in [BLPS that

$$
l_{-}^{2}(\Gamma)=\star \oplus \diamond \oplus \nabla H D
$$

where $\nabla H D$ is the gradient of the space of all harmonic Dirichlet functions on $\Gamma$. It is proven in [BLPS] that the distributions of WSF and FSF are the determinantal probability measures associated to $\star$ and $\star \oplus \nabla H D=\diamond^{\perp}$ respectively. To be 
precise, if $\vec{B}$ is a set of directed edges such that if $e \in \vec{B}$, then $\check{e} \notin \vec{B}$, then let $B$ denote the same set of edges of $\vec{B}$ but without orientation. Then we define $\mathbf{P}^{S}$ by

$$
\mathbf{P}^{S}(B \subset \mathfrak{B})=\operatorname{Det}\left[\left\langle P_{S}\left(\chi^{e}\right), \chi^{f}\right\rangle_{e, f \in \vec{B}}\right]
$$

where $S$ is a subspace of $l_{-}^{2}(\Gamma)$. It follows from elementary properties of deteminants that this is well defined (i.e., it is independent of the choice of $\vec{B}$ ). It is shown in BLPS that $\mathbf{P}^{\star}$ is the distribution of WSF and $\mathbf{P}^{\diamond^{\perp}}$ is the distribution of FSF. For our purposes, it suffices to assume this as the definition of the distributions of WSF and FSF. One easily deduces that WSF $=$ FSF if and only if $\nabla H D=0$. This occurs, for example, when $\Gamma$ is the Cayley graph of an amenable group or of a Kazhdan group. See Bekka and Valette [BV] (Theorem D) for a more complete list of groups whose Cayley graphs satisfy $\nabla H D=0$.

We will need the following facts from [L2] (Theorem 5.2). If $S<T<l_{-}^{2}(\Gamma)$ and $S$ and $T$ are closed, then for any increasing event $\mathcal{A} \subset 2^{E(\Gamma)}(\mathcal{A}$ is increasing whenever $A \in A$ and $A \subset B \subset E(\Gamma)$ implies $B \in \mathcal{A}), \mathbf{P}^{S}(\mathcal{A}) \leq \mathbf{P}^{T}(\mathcal{A})$. By Strassen's theorem [St], this implies the existence of a monotone coupling between $\mathbf{P}^{S}$ and $\mathbf{P}^{T}$.

\section{THE THEOREM}

In this section we state and prove the main theorem.

Theorem 4.1. If $\Gamma$ is a connected locally finite graph (with at most a countable number of vertices) and $G<\operatorname{Aut}(\Gamma)$ is residually amenable (with respect to the compact-open topology), then there exists a G-invariant monotone coupling between the WSF and the FSF on $\Gamma$.

Since $G$ is residually amenable and separable, there exists a decreasing sequence $\left\{G_{i}\right\}$ of closed normal subgroups of $G$ such that $G / G_{i}$ is amenable for all $i$ and $\bigcap_{i} G_{i}=\{1\}$.

We let $\Gamma / G_{i}$ be the quotient graph. Its vertices (edges) are equivalence classes of vertices (edges) in $\Gamma$ where $v$ is equivalent to $w$ if there is a $g \in G_{i}$ such that $g v=w$. If $[v]$ and $[w]$ are vertices of $\Gamma / G_{i}$, then there is an edge between them for every equivalence class $[e]$ of edges such that one endpoint of $e$ is in $[v]$ and the other is in $[w]$. Consider $l_{-}^{2}\left(\Gamma / G_{i}\right)$. We let $\star_{i}$ be the subspace generated by all the stars of $\Gamma / G_{i}$ and $\diamond_{i}$ be the subspace generated by all the cycles of $\Gamma / G_{i}$. We let $\nabla H D_{i}$ denote the gradient of the harmonic Dirichlet functions on $\Gamma / G_{i}$. So,

$$
l_{-}^{2}\left(\Gamma / G_{i}\right)=\star_{i} \oplus \diamond_{i} \oplus \nabla H D_{i} .
$$

Let $\pi_{i}: \Gamma \rightarrow \Gamma / G_{i}$ denote the quotient map. Suppose $c$ is a cycle in $l_{-}^{2}\left(\Gamma / G_{i}\right)$. Then $c=\sum_{i=1}^{n} \chi^{e_{i}}$ where $e_{1}, e_{2}, \ldots, e_{n}$ is a sequence of directed edges such that the terminal vertex of $e_{i}$ is equal to the initial vertex of $e_{i+1}(\bmod n)$. We will say that $c$ is a true cycle if every directed component of $\pi_{i}^{-1}\left(\left\{e_{1}, \ldots, e_{n}\right\}\right)$ forms a (finite) cycle in $\Gamma$. We let $C_{i}$ denote the closed subspace of $\diamond_{i}$ spanned by all the true cycles and let $H_{i}$ denote its orthocomplement in $\diamond_{i}$. Now we have

$$
l_{-}^{2}\left(\Gamma / G_{i}\right)=\star_{i} \oplus C_{i} \oplus H_{i} \oplus \nabla H D_{i} .
$$

Now let $W_{i}$ be the random subgraph of $\Gamma / G_{i}$ associated to $\star_{i}$. Let $F_{i}$ be the random subgraph associated to $\star_{i} \oplus H_{i} \oplus \nabla H D_{i}=C_{i}^{\perp}$.

The strategy of the proof is as follows. We show that in a certain sense, a lift of $W_{i}$ converges to WSF and a lift of $F_{i}$ converges to FSF. Using the amenability 
of $G / G_{i}$, we can average any coupling of $W_{i}$ and $F_{i}$ over $G / G_{i}$ to obtain a $G / G_{i^{-}}$ invariant coupling. By a compactness argument, a limit point of the sequence of lifted couplings exists and we will show that any limit point is a $G$-invariant monotone coupling of WSF and FSF.

We need to introduce some topological spaces. First, we let $Z=2^{E(\Gamma)}$ be the space of subgraphs of $\Gamma$. It is compact in the product topology. The group $G$ acts on $Z$ in the natural way, and this action is bi-continuous. We let $M_{Z}$ denote the set of Borel probability measures on $Z$, and let $M_{Z}^{i}$ denote the subset of $M_{Z}$ of measures that are $G$-invariant. By invariance we mean if $\mu \in M_{Z}^{i}, E \subset Z$ and $g \in G$, then $\mu(g E)=\mu(E)$. We say that a sequence $\left\{\mu_{i}\right\}$ in $M_{Z}$ converges to $\mu$ in the weak* topology if

$$
\int_{Z} f d \mu_{i} \rightarrow \int_{Z} f d \mu
$$

for every continuous function $f: Z \rightarrow \mathbb{C}$. It follows from standard functional analysis that both $M_{Z}$ and $M_{Z}^{i}$ are compact under the weak* topology. Note that for every finite subset $B \subset E(\Gamma)$, the function $f_{B}: Z \rightarrow \mathbb{C}$ defined by $f_{B}(z)=1$ if $B \subset z$ and $f_{B}(z)=0$ otherwise is continuous. It is not hard to show that if

$$
\int_{Z} f_{B} d \mu_{i} \rightarrow \int_{Z} f_{B} d \mu
$$

for every finite set $B \subset E(\Gamma)$, then $\mu_{i}$ converges to $\mu$ in the weak* topology. In this case, if $\mathcal{G}_{i}$ is a random subgraph with distribution $\mu_{i}$ and $\mathcal{G}$ is a random subgraph with distribution $\mu$, then we will say that $\mathcal{G}_{i}$ converges to $\mathcal{G}$ (weak*). Similarly, we define $M_{Z \times Z}^{i}$ to be the space of $G$-invariant Borel probablity measures on $Z \times Z$. It is compact under the weak* topology.

If $\left\{S_{i}\right\}$ is a sequence of closed subspaces in $l_{-}^{2}(\Gamma)$, we will say that $P_{S_{i}} \rightarrow P_{S}$ in the strong operator topology (SOT) if for every $f \in l_{-}^{2}(\Gamma),\left\|P_{S_{i}}(f)-P_{S}(f)\right\| \rightarrow 0$. We sometimes express this by writing $S_{i} \rightarrow S$ (SOT). It can be shown that the set of subspaces of $l_{-}^{2}(\Gamma)$ is compact under the SOT. Note that if $P_{S_{i}} \rightarrow P_{S}$ (SOT), then $\mathbf{P}^{S_{i}} \rightarrow \mathbf{P}^{S}$ in the weak* topology. It is well known that if $S_{i}<l_{-}^{2}(\Gamma)$ are closed subspaces and $S_{i} \nearrow S$ (meaning that $S_{i} \subset S_{i+1}$ and $\bigcup S_{i}$ is dense in $S$ ), then $P_{S_{i}}(T) \rightarrow P_{S}(T)$ (SOT) for any closed subspace $T$ (in $l_{-}^{2}(\Gamma)$ ).

If $S<l_{-}^{2}\left(\Gamma / G_{i}\right)$, let $\tilde{\mathbf{P}}^{S}$ be the distribution of the random subgraph $\pi_{i}^{-1}\left(\mathcal{G}_{S}\right)$. For ease of notation, we also denote $\pi_{i}^{-1}\left(W_{i}\right)$ by $\tilde{W}_{i}$ and $\pi_{i}^{-1}\left(F_{i}\right)$ by $\tilde{F}_{i}$. We will show that $\tilde{W}_{i} \rightarrow \mathrm{WSF}$ and $\tilde{F}_{i} \rightarrow \mathrm{FSF}$. In order to do this, we introduce sequences of random subgraphs of $\Gamma$ that equal $\tilde{W}_{i}$ or $\tilde{F}_{i}$ on fundamental domains of $G_{i}$ and have the advantage that their distributions are determinantal. For this we let $D_{i}$ be a connected subgraph of $\Gamma$ such that the covering map $\pi_{i}$ restricted to the edge set of $D_{i}$ is bijective. Also, assume that $D_{i} \subset D_{i+1}$ and that $\bigcup_{i} D_{i}=\Gamma$. This is possible since $\bigcap_{i} G_{i}=\{1\}$.

If $\mathcal{G}_{S}$ is a random subgraph of $\Gamma / G_{i}$, let $\widehat{\mathcal{G}_{S}}$ be the random subgraph of $\Gamma$ that is contained in $D_{i}$ and is equal to $\widetilde{\mathcal{G}_{S}}$ on $D_{i}$. Note that $\pi_{i}$ restricted to $D_{i}$ induces an isomorphism $\pi_{i *}: l_{-}^{2}\left(D_{i}\right) \rightarrow l_{-}^{2}\left(\Gamma / G_{i}\right)$. If $S<l_{-}^{2}\left(\Gamma / G_{i}\right)$ is a (closed) subspace, let $\hat{S}$ denote $\pi_{i *}^{-1}(S)$. It is easy to check that $\hat{\mathcal{G}}_{S}$ is the random subgraph associated to $\hat{S}$ (i.e., $\hat{\mathcal{G}}_{S}=\mathcal{G}_{\hat{S}}$ ).

Lemma 4.2. Suppose for each $i$ that $\mathcal{G}_{i}$ is a random subgraph on $\Gamma / G_{i}$. Then $\tilde{\mathcal{G}}_{i} \rightarrow \mathcal{G}\left(\right.$ weak $\left.^{*}\right)$ if and only if $\hat{\mathcal{G}}_{i} \rightarrow \mathcal{G}\left(\right.$ weak $\left.^{*}\right)$. 
Proof. Let $B$ be a finite set of edges of $\Gamma$. Suppose that $\tilde{\mathcal{G}}_{i} \rightarrow \mathcal{G}$ (weak*). Then, by definition of weak* convergence,

$$
\int_{Z} f_{B} d \tilde{\mu}_{i} \rightarrow \int_{Z} f_{B} d \mu
$$

where $\tilde{\mu}_{i}$ is the distribution of $\tilde{\mathcal{G}}_{i}$ and $\mu$ is the distribution of $\mathcal{G}$. There exists an $N$ such that $i>N$ implies that $B \subset D_{i}$. Since $\hat{\mathcal{G}}_{i}$ is equal to $\tilde{G}_{i}$ on $D_{i}$, it follows that

$$
\int_{Z} f_{B} d \hat{\mu}_{i} \rightarrow \int_{Z} f_{B} d \mu
$$

where $\hat{\mu}_{i}$ is the distribution of $\hat{\mathcal{G}}_{i}$. Since $B$ is arbitrary, $\hat{\mathcal{G}}_{i} \rightarrow \mathcal{G}$ (weak*). The proof in the other direction is similar.

Theorem 4.3. $\tilde{W}_{i} \rightarrow$ WSF and $\tilde{F}_{i} \rightarrow$ FSF $\left(\right.$ weak $\left.^{*}\right)$.

Proof. By the above lemma, it suffices to prove that $\hat{W}_{j} \rightarrow \mathrm{WSF}$ and $\hat{F}_{j}$ to FSF. To this end, it suffices to prove that $\hat{\star}_{j} \rightarrow \star$ and $\widehat{C_{j}^{\perp}} \rightarrow \diamond^{\perp}$. Let $B$ be a finite set of (directed) edges of $\Gamma$, and let $S$ be the subspace generated by $\chi^{e}$ for $e \in B$.

We show first that $P_{S}(\star)<P_{S}\left(\hat{\star}_{j}\right)$ for all large enough $j$. Suppose that $v$ is a vertex incident to an edge $e \in B$. Let $f_{v}$ be the star of $v$. It suffices to show that $P_{S}\left(f_{v}\right) \in P_{S}\left(\hat{\star}_{j}\right)$ for all large enough $j$. So let $j$ be large enough so that all edges incident to $v$ are in $D_{j}$. Let $g_{v}$ be the star of $\pi_{j}(v)$ in $\Gamma / G_{j}$. By definition $g_{v} \in \star_{j}$. It is clear that $f_{v}=\pi_{j *}^{-1}\left(g_{v}\right)$. So $P_{S}\left(f_{v}\right)=P_{S}\left(\pi_{j *}^{-1}\left(g_{v}\right)\right)$, which implies that $P_{S}\left(f_{v}\right) \in P_{S}\left(\hat{\star}_{j}\right)$. Since $v$ is arbitrary, the claim is proven.

To show that $P_{S}\left(\hat{\star}_{j}\right)<P_{S}(\star)$ for $j$ large enough we follow the same argument as above in reverse. So, $P_{S}\left(\hat{\star}_{j}\right)=P_{S}(\star)$ for all $j$ large enough. Similarly, it can be shown that $P_{S}\left(\hat{C}_{j}\right)=P_{S}(\diamond)$ for all $j$ large enough.

We let $S_{i}$ be the subspace generated by the functions $\chi^{e}$ for $e \in D_{i}$. Then $S_{i} \nearrow l_{-}^{2}(\Gamma)$; so $P_{S_{i}}(\star) \rightarrow \star(\mathrm{SOT})$. Since $P_{S_{i}}(\star)=P_{S_{i}}\left(\hat{\star}_{j}\right)$ for $j$ large enough, we have that for any subsequential limit $T$ for $\left\{\hat{\star}_{j}\right\}, P_{S_{i}}(T) \rightarrow \star$ (SOT). But $P_{S_{i}}(T) \rightarrow T(\mathrm{SOT})$; so $T=\star$, i.e., $\hat{\star}_{j} \rightarrow \star$ (SOT). This implies that $\hat{W}_{i} \rightarrow$ WSF (weak*).

Similarly, it can be shown that $\hat{C}_{i} \rightarrow \diamond(\mathrm{SOT})$. This implies that $\left(\hat{C}_{i}\right)^{\perp} \rightarrow$ $\diamond^{\perp}(\mathrm{SOT})$. Since $S_{i} \nearrow l_{-}^{2}(\Gamma)$, it follows that $P_{S_{i}}\left(\left(\hat{C}_{i}\right)^{\perp}\right) \rightarrow \diamond^{\perp}$. But note that $P_{S_{i}}\left(\left(\hat{C}_{i}\right)^{\perp}\right)=\widehat{C_{i}^{\perp}}$. Hence we have shown that $\widehat{C_{i}^{\perp}} \rightarrow \diamond^{\perp}$. This implies that $\hat{F}_{i} \rightarrow$ FSF $\left(\right.$ weak $\left.^{*}\right)$ since $\widehat{C_{i}^{\perp}}$ is the distribution of $\hat{F}_{i}$. Now we are done.

Proof of Theorem 4.1. By Strassen's lemma and [L2, Theorem 6.1, for all $i$, there exists a monotone coupling of $W_{i}$ and $F_{i}$. The space $\mathcal{C}_{i}$ of all such monotone couplings is compact under the weak* topology. $G / G_{i}$ acts on this space by $g G_{i} \mu(F) \rightarrow \mu\left(g G_{i} F\right)$ for $\mu \in \mathcal{C}_{i}$ and $F \subset 2^{E\left(\Gamma / G_{i}\right)} \times 2^{E\left(\Gamma / G_{i}\right)}$. Since $G / G_{i}$ is amenable, there exists a $G / G_{i}$-invariant measure $\sigma_{i}$ on $\mathcal{C}_{i}$. Let $\mu_{i}$ be defined by

$$
\mu_{i}(F)=\int_{\mathcal{C}_{i}} \nu(F) d \sigma_{i}(\nu)
$$

for any Borel set $F \subset 2^{E\left(\Gamma / G_{i}\right)}$. Since $\sigma_{i}$ is $G / G_{i}$-invariant, $\mu_{i}$ is a $G / G_{i}$-invariant monotone coupling of $W_{i}$ and $F_{i}$. 
Since $\pi_{i}: \Gamma \rightarrow \Gamma / G_{i}$ is equivariant with respect to the $G$-action, and $\mu_{i}$ is invariant under $G / G_{i}$, we may pull $\mu_{i}$ back via $\pi_{i}$ to a $G$-invariant monotone coupling $\tilde{\mu}_{i}$ between $\tilde{W}_{i}$ and $\tilde{F}_{i}$.

The space of $G$-invariant monotone couplings is naturally a closed subspace of $M_{Z \times Z}^{i}$. This space is compact. So there exists a weak* limit point $\mu$ of $\tilde{\mu}_{i}$. Since $\mu_{i} \rightarrow \mu$, the first and second marginals of $\mu_{i}$ must converge to the first and second marginals of $\mu$ respectively. Thus $\mu$ is a coupling between WSF and FSF.

\section{Speculations}

(1) Suppose that $\Gamma$ is a planar graph embedded in the hyperbolic plane so that $\operatorname{Aut}(\Gamma)$ acts by hyperbolic isometries. We may then take $G=\operatorname{Aut}(\Gamma)$ and choose $G_{i}$ so that $G / G_{i}$ is finite. It can be shown that the distribution of $F_{i}$ is the uniform distribution on subgraphs $\mathcal{G}$ of $\Gamma / G_{i}$ such that $\mathcal{G}$ contains a spanning tree and $\mathbb{H}^{2} / G_{i}-\mathcal{G}$ is a topological disk. If there exists a "natural" or explicit coupling between $W_{i}$ and $F_{i}$, then one may hope to obtain a natural coupling between WSF and FSF as a limit of lifts of couplings of $W_{i}$ and $F_{i}$. It seems that it should be possible to find such a coupling (if it exists) if only because $\Gamma / G_{i}$ is a finite graph.

(2) Suppose that $S$ is a $G$-invariant subspace of $l_{-}^{2}(\Gamma)$. Do there exist $G / G_{i^{-}}$ invariant subspaces $S_{i}$ in $l_{-}^{2}\left(\Gamma / G_{i}\right)$ such that $\hat{S}_{i} \rightarrow S$ ?

\section{ACKNOWLEDGMENTS}

I am grateful to Henry Cohn, Scott Sheffield, Russell Lyons and Yuval Peres for valuable conversations.

\section{REFERENCES}

[BLPS] I. Benjamini, R. Lyons, Y. Peres, and O. Schramm, Uniform Spanning Forests, Ann. Probab. 29 (2001), no. 1, 1-65. MR 2003a:60015

[BV] M. E. B. Bekka and A. Valette, Group cohomology, harmonic functions and the first $L^{2}$-Betti number, Potential Anal. 6 (1997), 313-326. MR 98e:20056]

[FM] T. Feder and M. Mihail, Balanced Matroids, Proc. 24th Annual ACM Sympos. Theory Computing (Victoria, BC, Canada), pp. 26-38, ACM Press, New York, 1992.

[L1] R. Lyons, A bird's-eye view of uniform spanning trees and forests, in Microsurveys in Discrete Probability, D. Aldous and J. Propp (eds.), Amer. Math. Soc., Providence, RI, 1998, pp. 135-162. MR 99e:60029

[L2] R. Lyons, Determinantal Probability Measures, preprint.

[KW] Ilya Kapovich and Daniel T. Wise, The equivalence of some residual properties of wordhyperbolic groups, J. Algebra 223 (2000), no. 2, 562-583. MR 2001f:20086

[Ma] A. I. Mal'cev, On isomorphic matrix representations of infinite groups (Russian). Mat. Sbornik 8, 405-422 (1940); Amer. Math. Soc. Transl. (2) 45, 1-18 (1965). MR 2:216d

[O] A. Yu. Olshanskii, Almost every group is hyperbolic, Internat. J. Algebra Comput. 2 (1992), no. 1, 1-17. MR 93j:20068

[Pe] Robert Pemantle, Choosing a spanning tree for the integer lattice uniformly, Ann. Probab. 19 (1991), 1559-1574. MR 92g:60014

[Pi] Jean-Paul Pier, Amenable locally compact groups, Pure and Applied Mathematics. A Wiley-Interscience Publication. John Wiley \& Sons, Inc., New York, 1984. x+418 pp. MR 86a:43001

[S] E. A. Scott, A tour around finitely presented infinite simple groups, Algorithms and classification in combinatorial group theory (Berkeley, 1989), pp. 83-119, Springer-Verlag, New York, 1992. MR 94j:20030

[St] V. Strassen, The existence of probability measures with given marginals, Ann. Math. Statist. 36 (1965), 423-439. MR 31:1693 
[We] B. A. F. Wehrfritz, Infinite Linear Groups, An account of the group-theoretic properties of infinite groups of matrices, Ergebnisse der Matematik und ihrer Grenzgebiete, Band 76, Springer-Verlag, New York, Heidelberg, Berlin, 1973. MR 49:436

[Z] Robert J. Zimmer, Ergodic theory and semisimple groups, Monographs in Mathematics, 81. Birkhäuser Verlag, Basel, 1984. x+209 pp. MR 86j:22014

Department of Mathematics, University of California, Davis, California 95616

E-mail address: lbowen@math.ucdavis.edu 